\title{
Level of Endogenous Jasmonate is Critical for Durable Broad-Spectrum Disease Resistance Against Rice Blast in a Japonica Rice Variety, Ziyu44
}

\section{Xingyu An}

Yunnan Agricultural University

Hui Zhang

Yunnan Agricultural University

Jinlu Li

Yunnan Agricultural University

Rui Yang

Yunnan Agricultural University

Qianchun Zeng

Yunnan Agricultural University

Mo Wang

Fujian Agriculture and Forestry University

Qiong Luo ( $\nabla$ qiongbf@aliyun.com )

Yunnan Agricultural University

\section{Research Article}

Keywords: rice, blast disease, durable blast resistance, Ziyu44, JA, SA

Posted Date: June 24th, 2021

DOI: https://doi.org/10.21203/rs.3.rs-571828/v1

License: (9) This work is licensed under a Creative Commons Attribution 4.0 International License.

Read Full License 


\section{Abstract}

Background: The molecular mechanism of durable and broad-spectrum resistance to rice blast disease in japonica rice variety is still very little known. Ziyu44, a local japonica rice variety in Yunnan Province of China, has shown durable broad-spectrum blast resistance for more than 30 years, and provides an opportunity for us to explore the molecular basis of broad-spectrum resistance to rice blast in japonica rice variety.

Methods and Results: We conducted a comparative study of mycelium growth, aposporium formation, the accumulation of salicylate(SA), jasmonate(JA) and $\mathrm{H}_{2} \mathrm{O}_{2}$, the expression of SA- and JA-associated genes between Ziyu44 and susceptible variety Jiangnanxiangnuo (JNXN) upon M. oryzae infection. We found that appressorium formation and invasive hyphae extention were greatly inhibited in Ziyu 44 leaves compared with that in JNXN leaves. Both Ziyu 44 and JNXN plants maintained high levels of baseline SA and did not show increased accumulation of SA after inoculation with M. oryzae, while the levels of baseline JA in Ziyu 44 and JNXN plants were relatively low, and the accumulation of JA exhibited markedly increased in Ziyu 44 plants upon M. oryzae infection. The expression levels of key genes involving JA and SA signaling pathway OsCOI1b, OsNPR1, OsMPK6 as well as pathogenesisrelated (PR) genes OsPR1a, OsPR1b and OsPBZ1, were markedly up-regulated in Ziyu44.

Conclusions: The level of endogenous JA is critical for synchronous activation of SA and JA signaling pathway, up-regulating PR gene expression and enhancing disease resistance against rice blast in Ziyu44.

\section{Introduction}

Rice blast, caused by the fungus Maganaporthe oryzae, is the most devastating rice disease resulting in the worldwide annual loss of $10-30 \%$ of the rice harvest, amount enough to feed approximately 60 million people. Deployment of resistance $(R)$ genes in rice is considered as the best practice to manage diseases and curtail the environmental damage by reducing the use of agro-chemicals[1]. To date, more than $100 \mathrm{R}$ genes have been identified in rice, and 25 of them have been cloned and characterized[2]. Although some $R$ genes conferring strain-specific resistance have been applied in plant breeding, their effect can be rapidly overcome by the emergence of compatible blast isolates[1]. In addition, because pyramiding $R$ genes to develop resistant cultivars is an extremely time-consuming process, rice production is still facing a huge threat caused by the fast evolution of pathogenic blast fungi[3]. Therefore, characterizing the molecular mechanism of durable and broad-spectrum resistance is important for guiding rice resistance breeding. Gumei 4 (GM4) and Digu, two Chinese Indica rice varieties, display high and durable blast resistance[4, 5]. Recent studies have shown that PigmR gene encoding nucleotide-binding leucine-rich repeat (NLR) receptor confers broad-spectrum resistance in GM4[4], and a single base change (SNP33-G) in the bsr-d1 (a natural allele of a $\mathrm{C} 2 \mathrm{H} 2$-domain transcription factor gene) promoter enhances binding to MYBS1 and confers broad-spectrum resistance to rice blast in Digu[5]. 
The cultivated rice (Oryza sativa L.) is divided into two main subspecies: Japonica and indica. Because japonica rice has better taste quality than indica rice, the planting area in China is expanding year by year. However, compared with indica rice, the blast resistance of japonica rice is generally very poor[6]. In fact, most of the identified broad-spectrum blast resistance resources are indica rice, like Digu and Gumei, and the molecular mechanisms on broad-spectrum blast resistance characterized so far are limited to indica rice and these genes cloned from ndica rice materials. However, the resistance resources of japonica rice, especially those with broad-spectrum blast resistance, are relatively scarce, and the broad-spectrum resistance mechanism of japonica rice is still very little known, which is very unfavorable to the overall understanding of the molecular mechanism of broad-spectrum resistance to rice blast.

Plants employ a two-tier innate immunity system to protect them from a wide range of pathogens: pathogen-associated molecular pattern (PAMP)-triggered immunity (PTI), and effector-triggered immunity $(\mathrm{ETI})[7,8]$. PTI contributes to host defense against infections by a broad range of pathogens, activation of PTI leads to various immune responses, including calcium influx, the deposition of callose, a rapid burst of reactive oxygen species (ROS), and expression of defense genes[9]. These responses confer effective and broad-spectrum defense against the majority of potential pathogens. Downstream of PTI activation, the activation of complex phytohormones signaling networks is critical for stimulating plant innate immunity[10]. Salicylic acid (SA) and jasmonate (JA) are recognized as the most important hormones in plant immune responses and are believed to represent the hormonal backbone of defense against pathogens[11]. In general, the SA pathway is crucial for immune responses against biotrophic and hemibiotrophic pathogens, whereas the JA pathway is involved in defense against necrotrophic pathogens acquiring nutrients from the decaying host tissue[12, 13]. Moreover, interaction between these two types of defense is mostly antagonistic $[12,14,15]$. This reciprocal antagonistic crosstalk between the SA and JA pathways, initially demonstrated in Arabidopsis[16, 17], is present also in other plant species[18]. Nonetheless, evidence deviating of the antagonism between SA and JA also exists, particularly in monocotyledonous plants[19-22].

Ziyu44, a japonica rice variety of Yunnan Province, has broad-spectrum resistance to 16 physiological races (ZA1, ZA49, ZA57, ZA61, ZB1, ZB13, ZB17, ZB25, ZC1,ZC3, ZC13, ZC15, ZE1, ZE3, ZF1 and ZG1) from Yunnan province[23]. Over the past 30 years, field-cultivated Ziyu44 has displayed high and durable resistance to rice blast[24]. Our previous studies have identified a number of major and minor resistance genes in Ziyu44[24-26] and suggested that durable broad-spectrum resistance to rice blast in this cultivar may reflect a combined effect of multiple loci. However, the functions of SA and JA in regulating immunity in Ziyu44 are unclear. Specifically, the spatiotemporal dynamics of SA and JA during the interaction between Ziyu44 and $M$. oryzae, and the relative contribution of each hormone to the defense response of Ziyu44 remain unknown. Therefore, the objective of the present study was to compare appressorium formation, hypha growth, endogenous SA and JA content, and expression of SA- and JAassociated genes in Ziyu44 and JNXN rice varieties in response to $M$. oryzae infection. The obtained results revealed that the accumulation of JA and activation of the SA-JA defense signaling at the early stages of M. oryzae infection in the durably resistant rice Ziyu44 is essential for the resistance to rice blast. 


\section{Materials And Methods \\ Rice cultivars, fungal isolates}

Ziyu44 and Jiangnanxiangnuo (JNXN), two Chinese Japonica (Oryza sativa L. subsp. geng) rice varieties, used in this study. Ziyu44, a landrace of Yunnan Province, exhibits broad-spectrum and durable blast resistance, while the rice cultivar JNXN is susceptible to rice blast. The M. oryzae isolates of Zhong-10-814 (an M. oryzae strain tagged with enhanced green fluorescent protein (eGFP)) and LP33 were kindly provided by Prof. Lihuang Zhu (Institute of Genetics and Developmental Biology, Chinese Academy of Sciences) and Prof. Yueqiu He (Yunnan Agricultural University), respectively.

\section{Fungal growth on rice sheath}

The durably resistant rice Ziyu 44 and the susceptible rice JNXN were grown in a growth chamber at $28^{\circ} \mathrm{C}$ and $75 \%$ humidity in a $12 \mathrm{~h}$ light/ $12 \mathrm{~h}$ dark photoperiod. For microscope monitoring of fungal development, $M$. oryzae strains Zhong-10-8-14 and LP33 were grown on Potato sucrose medium for 2 weeks (at $26^{\circ} \mathrm{C}$ and natural light), then conidial spores were collected via flooding oat-tomato agar medium with sterile water. $5 \times 10^{5}$ conidia/ $\mathrm{mL}$ spore suspension were used to inoculate the detached rice sheaths from 4-week-old Ziyu44 and JNXN as described previously[27, 28]. The images of conidial germination, appressorium development, and invasive hyphae growth were recorded using an Olympus fluorescent microscope. The microscopic evaluation of the infected sheath was performed in three independent experiments, more than 30 cells were counted in each replicate.

\section{$\mathrm{H}_{2} \mathrm{O}_{2}$ accumulation}

Seedlings of Ziyu44 and JNXN rice with 3-4 leaves were spray-inoculated with M. oryzae strain LP33. The spore concentration in the suspension was adjusted to approximately $5 \times 10^{5}$ conidia/mL with $4 \%$ o gelatin. $\mathrm{H}_{2} \mathrm{O}_{2}$ accumulation was monitored by staining with $3,3^{\prime}$-diaminobenzidine (DAB, Sigma), as previously described[29]. Briefly, leaf sections were placed in $1 \mathrm{mg} \mathrm{mL}^{-1} \mathrm{DAB}$, incubated at $22^{\circ} \mathrm{C}$ for $10 \mathrm{~h}$ under illumination, and the formation of a brown precipitate of oxidized DAB was observed under a microscope.

\section{RNA isolation and qRT-PCR}

Total RNA was extracted using the MiniBEST Plant RNA Extraction Kit (TaKaRa, Dalian, China) according to the manufacturer's protocol. cDNA was synthesized using the ReverTra Ace Qpcr RT Master Mix with gDNA Remove kit (TOYOBO, Shanghai, China). The real-time quantitative reverse transcriptasepolymerase chain reaction (qRT-PCR) was conducted using a Bio-Rad (Hercules, CA, USA) CFX96 RealTime System coupled to a C1000 Thermal Cycler. The expression of the ubiquitin (Ubq) gene was used as a reference for the normalization of all qRT-PCR data[28]. The relative levels of gene expression were calculated using the $2^{-\Delta \Delta C T}$ method, and each determination was done in triplicate[30]. Sequences of the primers are listed in Table 1. 
Table 1

Primers used in the study.

\begin{tabular}{|lll|}
\hline Primer name & Forward sequence $\left(\mathbf{5}^{\prime}\right.$ to $\left.\mathbf{3}^{\prime}\right)$ & Reverse sequence $\left(\mathbf{5}^{\prime}\right.$ to $\mathbf{3}^{\prime} \mathbf{)}$ \\
\hline OsUbi & GCCCAAGAAGAGATCAAGAAC & AGATAACAACGGAAGCATAAAAGTC \\
\hline OsNPR1 & TGAGAGTCTACGAGGAAGTTGC & CGTTGTCTTTCAGGAGGTGGAT \\
\hline OsPAL1 & AGGAGCTCGGCTGCGTATT & ATGCCGAGGAACACCTTGTT \\
\hline OsAOS2 & CCCTAGCGTTGACAACAAGCA & CGGAGGTTGAAGCTTTGGTGA \\
\hline OsPR1a & TCGTATGCTATGCTACGTGTTT & CACTAAGCAAATACGGCTGACA \\
\hline OsPR1b & GGCAACTTCGTCGGACAGA & CCGTGGACCTGTTTACATTTT \\
\hline OsPBZ1 & CCCTGCCGAATACGCCTAA & CTCAAACGCCACGAGAATTTG \\
\hline
\end{tabular}

\section{$\mathrm{SA}, \mathrm{JA}$, and jasmonoyl-isoleucine (JA-lle) assays}

SA, JA, and JA-lle were prepared and quantified as previously described[31]. The levels of SA, JA and JAlle were measured by gas chromatography-mass spectrometry (GC-MS) using labeled internal standards. Three biological replicates were performed for the statistical analysis.

\section{Results}

\section{Response to M. oryzae infection in Ziyu44 and JNXN leaves}

The resistance response of rice plants to the $M$. oryzae infection is thought to be dependent on the stage of development of the fungus [32]. To characterize the resistance responses of Ziyu44 against blast fungus during early infection stages, we inoculated the leaf sheath of Ziyu44 with M. oryzae strains, Zhong 10-8-14 (tagged by eGFP) and LP33. JNXN, a susceptible variety, was used as a control. At the early invasion stage ( 3 hours post-infection, hpi), the conidial germination and germ tube extension of Zhong 10-8-14 and LP33 showed no obvious difference on the leaf sheaths of Ziyu44 and JNXN rice (Fig. 1). However, the development of $M$. oryzae was greatly affected at 10-48 hpi in the resistant Ziyu44 rice compared with the susceptible rice JNXN. Although appressorium formation was observed on both JNXN and Ziyu44 at $10 \mathrm{hpi}$, its frequency was approximately 10\% lower in Ziyu44 than in JNXN rice (Fig. 1). On JNXN, the invasive hyphae formed at $22 \mathrm{hpi}$ and extended to the neighboring cells at $34 \mathrm{hpi}$. In contrast, on Ziyu44, the invasive hyphae were mostly restricted to the primary infected leaf sheath cells at $34 \mathrm{hpi}$ and disappeared in some cells at $48 \mathrm{hpi}$ (Fig. 1). Additionally, extensive yellow autofluorescence was observed on Ziyu44. Therefore, we conclude that the appressorium formation, invasion hyphae growth and further extension were greatly inhibited in Ziyu44 leaves.

Accumulation of $\mathrm{H}_{2} \mathrm{O}_{2}$ in M. oryzae-infected leaf cells 
Reactive oxygen species (ROS) play important roles in both the first line of defense termed as pathogenassociated molecular patterns (PAMPs) triggered immunity (PTI) and the second line of defense related to effector-triggered immunity $(\mathrm{ETI})[33,34]$. To determine the generation of ROS during the infection, the leaves of the resistant Ziyu44 rice and the susceptible JNXN rice were inoculated with $M$. oryzae strain LP33 and stained with DAB. At 36 and 48 hpi, the infected leaf cells of Ziyu44 produced much darker staining than cells of JNXN, reflecting a higher concentration of $\mathrm{H}_{2} \mathrm{O}_{2}$ (Fig. 2). This result indicates that Ziyu44 may protect itself against the $M$. oryzae infection by enhancing the generation of ROS.

\section{The response of JA and SA in Ziyu44 and JNXN plants to M. oryzae infection}

Plant hormones, such as SA and JA, have conserved and divergent functions in fine-tuning immune responses in rice[21,35]. Typically, rice plants maintain a high level of free SA that is only weakly responsive to the pathogen attack[36]. Consistently with this report, our results showed that both Ziyu44 and JNXN plants maintained a high baseline levels of endogenous SA and did not show obvious changes of endogenous SA level after inoculation with M. oryzae (Fig. 3a). Although both Ziyu44 and JNXN plants maintained low levels of endogenous JA and jasmonoyl-isoleucine (JA-lle), the levels of endogenous JA and JA-lle were constitutively markedly increased only in Ziyu44 plants after inoculated with M. oryzae, which was 8.2 and $~ 20.2$ times higher than those in JNXN at 48 hpi, respectively (Fig. $3 \mathrm{~b}, \mathrm{c})$. These results indicate that increased accumulation of endogenous JA in Ziyu44 plants is important to mediate resistance to $M$. oryzae.

\section{Expression analysis of SA- and JA-associated genes}

To understand the roles of SA and JA signaling pathways in resistance of Ziyu44 agaist M. oryzae infection, the transcriptional levels of several SA- and JA-associated genes were examined using qRTPCR. OSPAL 1 encodes phenylalanine ammonia lyase, a key enzyme in the SA biosynthetic pathway[37], we observed that the expression levels of OSPAL 1 were low in both JNXN and Ziyu44 pants, and even down-regulated in Ziyu44 pants inoculated with M. oryzae LP33 at $24 \mathrm{hpi}(\mathrm{Fig} .4 \mathrm{a})$. OsAOS2 encodes allene oxide synthase which is a key enzyme in the JA biosynthetic pathway[38], the transcriptions of OsAOS2 were significantly up-regulated in Ziyu44 and JNXN after inoculation with M. oryzae LP33 (Fig. 4b). The expression patterns of OSAOS2 and OSPAL 1 were consistent with the accumulation of endogenous SA (Fig. 3a) and JA (Fig. 3b) in Ziyu44 and JNXN plants upon M. oryzae infection.

OsCOI1b is a JA receptor and play key roles in JA signaling[39], OsNPR1 is a key regulator of SA signaling pathway[40], and OsCOI1-mediated JA pathway is indispensable for the disease resistance conferred by OsNPR1[21]. OsMPK6 is a major MAP kinase of SA pathway[41]. OsCOI1b, OsNPR1 and OsMPK6, as well as PATHOGENESIS-RELATED (PR) genes, such as OsPR1a[42], OsPR1b and OSPBZ1[43], play a fundamental role in a plant's response to pathogen challenge. So we measured the expression of OsNPR1, OsMPK6, OsCOI1b, OsPR1a, OsPR10a and OsPR1b six genes in Ziyu44 and JNXN plants challenged with $M$. oryzae LP33. We observed that the expression levels of these six genes are remarkably up-regulated in both varieties plants at $24 \mathrm{hpi}$, the expression levels of them were significantly 
higher in resistant rice Ziyu44 plants than that in susceptible JNXN plants (Fig. 4). Therefore, we conclude that the increased accumulation of endogenous JA and synchronous activation of SA and JA signaling pathways may be critical in mediating rice Ziyu44 defense responses to $M$. oryzae infection.

\section{Discussion}

Ziyu44, a japonica rice variety of Yunnan Province, has exhibited durable broad-spectrum resistance to rice blast for over 30 years. Previous studies have identified multiple major and minor blast resistance genes and suggested that their combination underlies the broad-spectrum and durable resistance of this cultivar[24-26]. According to the resistance manifestations, the host's resistance to pathogens can be divided into resistance to contact, resistance to infection, and resistance to spread. Although $24 \mathrm{hpi}$ is considered the critical point in M. oryzae invasion[44], the significant differences of $M$. oryzae development between Ziyu44 and JNXN was oberserved as early as $10 \mathrm{hpi}($ Fig. 1b). At this developmental stage, the tip of the germ tube differentiated into a dome-shaped structure called the appressorium (Fig. 1a). Invasive hyphae formed on JNXN at $22 \mathrm{hpi}$ and spread to neighboring cells at 34 hpi. In contrast, on Ziyu44, invasive hyphae were still restricted in the primary infected cells at $34 \mathrm{hpi}$ and disappeared in some cells at $48 \mathrm{hpi}$ (Fig. 1a). We suggest that a series of early defense responses in Ziyu44 inhibit key aspects of $M$. oryzae development, including the formation and maturation of appressorium, primary infection, and the extension of invasive hyphae, which play important roles in conferring durable resistance to $M$. oryzae on Ziyu44.

Production of reactive oxygen species (ROS) during PTI occurs is critical for successful activation of immune responses against pathogen infection [45]. Rice plants modulate their activities of ROS generating and scavenging enzymes, mainly on NADPH oxidase OsRbohB, by different signaling pathways to accumulate ROS against rice blast [34]. Treating rice plants with SA or methyl-jasmonate (MeJA) can induce ROS accumulation and enhance the resistance against rice blast[46, 47]. The current work documented that Ziyu44 and JNXN plants contain low baseline levels of JA, and that the levels of JA and JA-lle are dramatically increased after inoculation with $M$. oryzae in Ziyu44 compared with JNXN (Fig. 3b and 3c), the production of $\mathrm{H}_{2} \mathrm{O}_{2}$ was also higher in the inoculated leaf cells of Ziyu44 than JNXN, resulting in darker DAB staining at 36 and $48 \mathrm{hpi}$ (Fig. 2), indicating that ROS play an important role in inhibiting the extension of invasive hyphae in Ziyu44. The autofluorescence of rice cells is considered to be associated with plant resistance to rice blast [48]. It is also notable that autofluorescence was also visible in leaf cells of Ziyu44 inoculated with M. oryzae Zhong-10-8-14 (a GFP-tagged M. oryzae strain) at 22-48 hpi (Fig. 1a). This is probably because the infection of $M$. oryzae caused the accumulation of callose in the cell wall of rice Ziyu44. This speculation needs further experimental verification.

Phytohormones are small molecules produced by plants that govern diverse physiological processes, including the defense against pathogens. Among them, SA, JA, and ethylene (ET) are the archetypal defense hormones and their importance in the hard wiring of the plant innate immune system is well 
documented in the model plant Arabidopsis thaliana, suggesting that plant innate immunity follows a binary model with SA and JA-ET having opposing influences[12, 14, 18]. In contrast to the simple binary SA versus JA-ET defense model in A. thaliana, disease resistance in rice appears to be controlled by a more complicated signaling network that does not support a dichotomy between the effectiveness of the SA, JA, and ET pathways and the lifestyle of a given pathogen[19], JA plays a significant role in $P R$ gene induction and defence against $M$. oryzae(a hemibiotrophic pathogen). Such as, exogenous application of JA was able to activate defense gene expression and local induced resistance in rice seedlings against the rice blast fungus[20, 38]; OsBAG4 is a BAG (Bcl-2-associated athanogene) family protein of rice, EBR1 (the rice E3 ubiquitin ligase) targets OsBAG4 and negatively regulates rice immunity, the transcript levels of many PR genes involved in rice PTI and the SA and JA pathways were decreased in OsBAG4RNAi protoplasts, while excessive accumulation of OsBAG4 triggers autoimmunity and enhances rice blast disease resistance in the ebr1 mutant or OsBAG4-OX (plants overexpressing OsBAG4-Flag), SA and JA signals were synchronously magnified in both of these lines[49]; Mitogen-activated protein kinase (MAPK) cascades play central roles in response to biotic and abiotic stresses. The mpk15 (knock-out mutant of OSMPK15) mutant lines exhibited an increased resistance to $M$. oryzae, phytohormones SA and JA were accumulated, and the expression of SA- and JA-pathway associated genes were significantly upregulated in mpk 15 mutant rice[50]. In the present study, we observed increased accumulation of endogenous JA, OsCOI1b (encods a JA receptor), OsNPR1(encodes a key regulator of SA-signaling pathway), OsMPK6 (encodes a major MAP kinase of SA pathway), as well as a number of PR genes OsPR1a, OsPR1b and OSPBZ1 were markedly up-regulated in resistant rice Ziyu44 upon $M$. oryzae infection, implying the endogenous JA level may be critical in mediating rice Ziyu44 defense responses to $M$. oryzae infection. To increase the level of endogenous JA by genetic manipulation may be effective in improving the resistance to rice blast in japonica rice varieties.

\section{Declarations}

Author contributions $\mathrm{QL}$ was responsible for research design, funding acquisition and project administration. AXY and $\mathrm{HZ}$ conducted out the experiment. QL and AXY performed writing the manuscript and editing. JLL participated in PCR data analysis and drawings. RY participated in RNA isolation and qRT-PCR. QCZ had the idea for the article. MW critically revised the work. AXY and QL were responsible for interpretation of results. All authors read and approved the final manuscript, as well as in the final approval of the version to be published. AXY and $\mathrm{HZ}$ contributed equally.

Funding This study was supported by grants from the National Key Research and Development Program of China (2016YFD0100600), the National Science Foundation of China (31960568).

Consent to participate Not applicable.

Consent to publish Not applicable.

Conflict of interest The authors have no conflicts of interest to declare that are relevant to the content of this article. 


\section{References}

1. Skamnioti P, Gurr SJ (2009) Against the grain: safeguarding rice from rice blast disease. Trends in biotechnology 27:141-150.doi:10.1016/j.tibtech.2008.12.002

2. Li W, Chern M, Yin J, Wang J, Chen X (2019) Recent advances in broad-spectrum resistance to the rice blast disease. Current opinion in plant biology 50:114-120.doi:10.1016/j.pbi.2019.03.015

3. Dangl JL, Horvath DM, Staskawicz BJ (2013) Pivoting the plant immune system from dissection to deployment. Science 341:746-751.doi:10.1126/science.1236011

4. Deng Y, Zhai K, Xie Z, Yang D, Zhu X, Liu J, Wang X, Qin P, Yang Y, Zhang G et al (2017) Epigenetic regulation of antagonistic receptors confers rice blast resistance with yield balance. Science 355:962-965.doi:10.1126/science.aai8898

5. Li W, Zhu Z, Chern M, Yin J, Yang C, Ran L, Cheng M, He M, Wang K, Wang J et al (2017) A Natural Allele of a Transcription Factor in Rice Confers Broad-Spectrum Blast Resistance. Cell 170:114-126 e115.doi:10.1016/j.cell.2017.06.008

6. Shang J, Tao Y, Chen X, Zou Y, Lei C, Wang J, Li X, Zhao X, Zhang M, Lu Z et al (2009) Identification of a new rice blast resistance gene, Pid3, by genomewide comparison of paired nucleotide-binding site--leucine-rich repeat genes and their pseudogene alleles between the two sequenced rice genomes. Genetics 182:1303-1311.doi:10.1534/genetics.109.102871

7. Jones JD, Dangl JL (2006) The plant immune system. Nature 444:323-329.doi:10.1038/nature05286

8. Boller T, He SY (2009) Innate immunity in plants: an arms race between pattern recognition receptors in plants and effectors in microbial pathogens. Science 324:742-744.doi:10.1126/science.1171647

9. Dodds PN, Rathjen JP (2010) Plant immunity: towards an integrated view of plant-pathogen interactions. Nature reviews Genetics 11:539-548.doi:10.1038/nrg2812

10. Nejat N, Mantri N (2017) Plant Immune System: Crosstalk Between Responses to Biotic and Abiotic Stresses the Missing Link in Understanding Plant Defence. Current issues in molecular biology 23:116.doi:10.21775/cimb.023.001

11. Shigenaga AM, Berens ML, Tsuda K, Argueso CT (2017) Towards engineering of hormonal crosstalk in plant immunity. Current opinion in plant biology 38:164-172.doi:10.1016/j.pbi.2017.04.021

12. Thomma BP, Eggermont K, Penninckx IA, Mauch-Mani B, Vogelsang R, Cammue BP, Broekaert WF (1998) Separate jasmonate-dependent and salicylate-dependent defense-response pathways in Arabidopsis are essential for resistance to distinct microbial pathogens. Proceedings of the National Academy of Sciences of the United States of America 95:15107-

15111.doi:10.1073/pnas.95.25.15107

13. Glazebrook J (2005) Contrasting mechanisms of defense against biotrophic and necrotrophic pathogens. Annual review of phytopathology 43:205-

227.doi:10.1146/annurev.phyto.43.040204.135923

14. Robert-Seilaniantz A, Grant M, Jones JD (2011) Hormone crosstalk in plant disease and defense: more than just jasmonate-salicylate antagonism. Annual review of phytopathology 49:317- 
343.doi:10.1146/annurev-phyto-073009-114447

15. Thaler JS, Humphrey PT, Whiteman NK (2012) Evolution of jasmonate and salicylate signal crosstalk. Trends in plant science 17:260-270.doi:10.1016/j.tplants.2012.02.010

16. Penninckx IA, Eggermont K, Terras FR, Thomma BP, De Samblanx GW, Buchala A, Metraux JP, Manners JM, Broekaert WF (1996) Pathogen-induced systemic activation of a plant defensin gene in Arabidopsis follows a salicylic acid-independent pathway. The Plant cell 8:2309-

2323.doi:10.1105/tpc.8.12.2309

17. Penninckx IA, Thomma BP, Buchala A, Metraux JP, Broekaert WF (1998) Concomitant activation of jasmonate and ethylene response pathways is required for induction of a plant defensin gene in Arabidopsis. The Plant cell 10:2103-2113.doi:10.1105/tpc.10.12.2103

18. Berens ML, Berry HM, Mine A, Argueso CT, Tsuda K (2017) Evolution of Hormone Signaling Networks in Plant Defense. Annual review of phytopathology 55:401-425.doi:10.1146/annurev-phyto-080516035544

19. De Vleesschauwer D, Gheysen G, Hofte M (2013) Hormone defense networking in rice: tales from a different world. Trends in plant science 18:555-565.doi:10.1016/j.tplants.2013.07.002

20. Riemann M, Haga K, Shimizu T, Okada K, Ando S, Mochizuki S, Nishizawa Y, Yamanouchi U, Nick P, Yano $\mathrm{M}$ et al (2013) Identification of rice Allene Oxide Cyclase mutants and the function of jasmonate for defence against Magnaporthe oryzae. The Plant journal : for cell and molecular biology 74:226-238.doi:10.1111/tpj.12115

21. Yang DL, Yang Y, He Z (2013) Roles of plant hormones and their interplay in rice immunity. Molecular plant 6:675-685.doi:10.1093/mp/sst056

22. Ross A, Yamada K, Hiruma K, Yamashita-Yamada M, Lu X, Takano Y, Tsuda K, Saijo Y (2014) The Arabidopsis PEPR pathway couples local and systemic plant immunity. The EMBO journal 33:6275.doi:10.1002/embj.201284303

23. Zhang J, Hong R, Fan J, Zhang Y, Luo Q, Zeng Q (2011) Analysis of Broad Spectrum and Persistent Rice Blast Resistance in yunnan Local Rice Variety. Southwest China J Agric Sci 24:1323-1326(in Chinese with English abstract).doi:1310.3969/j.issn.1001-4829.2011.1304.1019

24. Zhuo X, Fan L, An X, Guo J, Luo Q (2019) Mapping of a new rice blast resistance gene in Ziyu 44, a rice landrace from Yunnan Province, China. Chinese Journal Rice Science 33:12-19(in Chinese with English abstract).doi:10.16819/j.1001-7216.2019.8054

25. Hu C, Liu J, Wang Y, Yang R, Wang B, He Y, Zeng Q, Luo Q (2017) Mapping of Pizy6(t), a Gene Conferring Resistance to the Rice Blast Strain LP11, in Oryza sativa subsp. japonica Cultivar Ziyu44. Chinese Bulletin of Botany 52:61-69(in Chinese with English abstract).doi:10.11983/CBB16126

26. Zhou R, Wang B, Yang R, Li S, Fan L, Zeng Q, Luo Q (2015) Quantitative Trait Loci Analysis of Rice Blast Resistance in Japonica Rice Variety Ziyu44. Chinese Bulletin of Botany 50:691-698(in Chinese with English abstract ).doi:10.11983/CBB15050

27. Mosquera G, Giraldo MC, Khang CH, Coughlan S, Valent B (2009) Interaction transcriptome analysis identifies Magnaporthe oryzae BAS1-4 as Biotrophy-associated secreted proteins in rice blast 
disease. The Plant cell 21:1273-1290.doi:10.1105/tpc.107.055228

28. Li W, Liu Y, Wang J, He M, Zhou X, Yang C, Yuan C, Wang J, Chern M, Yin J et al (2016) The durably resistant rice cultivar Digu activates defence gene expression before the full maturation of Magnaporthe oryzae appressorium. Molecular plant pathology 17:354-368.doi:10.1111/mpp.12286

29. Yin J, Zhu X, Yuan C, Wang J, Li W, Wang Y, He M, Cheng Q, Ye B, Chen W et al (2015) Characterization and fine mapping of a novel vegetative senescence lethal mutant locus in rice. Journal of genetics and genomics = Yi chuan xue bao 42:511-514.doi:10.1016/j.jgg.2015.05.005

30. Livak KJ, Schmittgen TD (2001) Analysis of relative gene expression data using real-time quantitative PCR and the 2(-Delta Delta C(T)) Method. Methods 25:402408.doi:10.1006/meth.2001.1262

31. Tong X, Qi J, Zhu X, Mao B, Zeng L, Wang B, Li Q, Zhou G, Xu X, Lou Y et al (2012) The rice hydroperoxide lyase OsHPL3 functions in defense responses by modulating the oxylipin pathway. The Plant journal : for cell and molecular biology 71:763-775.doi:10.1111/j.1365-313X.2012.05027.x

32. Iwai T, Seo S, Mitsuhara I, Ohashi Y (2007) Probenazole-induced accumulation of salicylic acid confers resistance to Magnaporthe grisea in adult rice plants. Plant \& cell physiology 48:915924.doi:10.1093/pcp/pcm062

33. Camejo D, Guzman-Cedeno A, Moreno A (2016) Reactive oxygen species, essential molecules, during plant-pathogen interactions. Plant physiology and biochemistry : PPB 103:1023.doi:10.1016/j.plaphy.2016.02.035

34. Kou Y, Qiu J, Tao Z (2019) Every Coin Has Two Sides: Reactive Oxygen Species during Rice(-)Magnaporthe oryzae Interaction. International journal of molecular sciences 20.doi:10.3390/ijms20051191

35. De Vleesschauwer D, Seifi HS, Filipe O, Haeck A, Huu SN, Demeestere K, Hofte M (2016) The DELLA Protein SLR1 Integrates and Amplifies Salicylic Acid- and Jasmonic Acid-Dependent Innate Immunity in Rice. Plant physiology 170:1831-1847.doi:10.1104/pp.15.01515

36. Silverman P, Seskar M, Kanter D, Schweizer P, Metraux JP, Raskin I (1995) Salicylic Acid in Rice (Biosynthesis, Conjugation, and Possible Role). Plant physiology 108:633639.doi:10.1104/pp.108.2.633

37. Zhou X, Liao H, Chern M, Yin J, Chen Y, Wang J, Zhu X, Chen Z, Yuan C, Zhao W et al (2018) Loss of function of a rice TPR-domain RNA-binding protein confers broad-spectrum disease resistance. Proceedings of the National Academy of Sciences of the United States of America 115:31743179.doi:10.1073/pnas.1705927115

38. Mei C, Qi M, Sheng G, Yang Y (2006) Inducible overexpression of a rice allene oxide synthase gene increases the endogenous jasmonic acid level, PR gene expression, and host resistance to fungal infection. Molecular plant-microbe interactions : MPMI 19:1127-1137.doi:10.1094/MPMI-19-1127

39. Lee HY, Seo JS, Cho JH, Jung H, Kim JK, Lee JS, Rhee S, Do Choi Y (2013) Oryza sativa COI homologues restore jasmonate signal transduction in Arabidopsis coi1-1 mutants. PloS one 8:e52802.doi:10.1371/journal.pone.0052802 
40. Yuan Y, Zhong S, Li Q, Zhu Z, Lou Y, Wang L, Wang J, Wang M, Li Q, Yang D et al (2007) Functional analysis of rice NPR1-like genes reveals that OsNPR1/NH1 is the rice orthologue conferring disease resistance with enhanced herbivore susceptibility. Plant biotechnology journal 5:313324.doi:10.1111/j.1467-7652.2007.00243.x

41. Ueno Y, Yoshida R, Kishi-Kaboshi M, Matsushita A, Jiang CJ, Goto S, Takahashi A, Hirochika H, Takatsuji H (2015) Abiotic Stresses Antagonize the Rice Defence Pathway through the TyrosineDephosphorylation of OsMPK6. PLoS pathogens 11:e1005231.doi:10.1371/journal.ppat.1005231

42. Agrawal GK, Jwa NS, Rakwal R (2000) A novel rice (Oryza sativa L.) acidic PR1 gene highly responsive to cut, phytohormones, and protein phosphatase inhibitors. Biochemical and biophysical research communications 274:157-165.doi:10.1006/bbrc.2000.3114

43. Wang D, Qin Y, Han J, Zhang L, Xu X, Liu X, Wang C, Liu X (2014) Expression analysis of innate immunity related genes in the true/field blast resistance gene-mediated defence response. Biotechnology, biotechnological equipment 28:999-1007.doi:10.1080/13102818.2014.978664

44. Talbot NJ (2003) On the trail of a cereal killer: Exploring the biology of Magnaporthe grisea. Annu Rev Microbiol 57:177-202.doi:10.1146/annurev.micro.57.030502.090957

45. Lee D, Lal NK, Lin ZD, Ma S, Liu J, Castro B, Toruno T, Dinesh-Kumar SP, Coaker G (2020) Regulation of reactive oxygen species during plant immunity through phosphorylation and ubiquitination of RBOHD. Nature communications 11:1838.doi:10.1038/s41467-020-15601-5

46. Li Y, Zhang Z, Nie Y, Zhang L, Wang Z (2012) Proteomic analysis of salicylic acid-induced resistance to Magnaporthe oryzae in susceptible and resistant rice. Proteomics 12:23402354.doi:10.1002/pmic.201200054

47. Li Y, Nie Y, Zhang Z, Ye Z, Zou X, Zhang L, Wang Z (2014) Comparative proteomic analysis of methyl jasmonate-induced defense responses in different rice cultivars. Proteomics 14:10881101.doi:10.1002/pmic.201300104

48. Takahashi A, Kawasaki T, Henmi K, Shi IK, Kodama O, Satoh H, Shimamoto K (1999) Lesion mimic mutants of rice with alterations in early signaling events of defense. The Plant journal : for cell and molecular biology 17:535-545.doi:10.1046/j.1365-313x.1999.00405.x

49. You Q, Zhai K, Yang D, Yang W, Wu J, Liu J, Pan W, Wang J, Zhu X, Jian Y et al (2016) An E3 Ubiquitin Ligase-BAG Protein Module Controls Plant Innate Immunity and Broad-Spectrum Disease Resistance. Cell host \& microbe 20:758-769.doi:10.1016/j.chom.2016.10.023

50. Hong Y, Liu Q, Cao Y, Zhang Y, Chen D, Lou X, Cheng S, Cao L (2019) The OsMPK15 Negatively Regulates Magnaporthe oryza and Xoo Disease Resistance via SA and JA Signaling Pathway in Rice. Frontiers in plant science 10:752.doi:10.3389/fpls.2019.00752

\section{Figures}


a

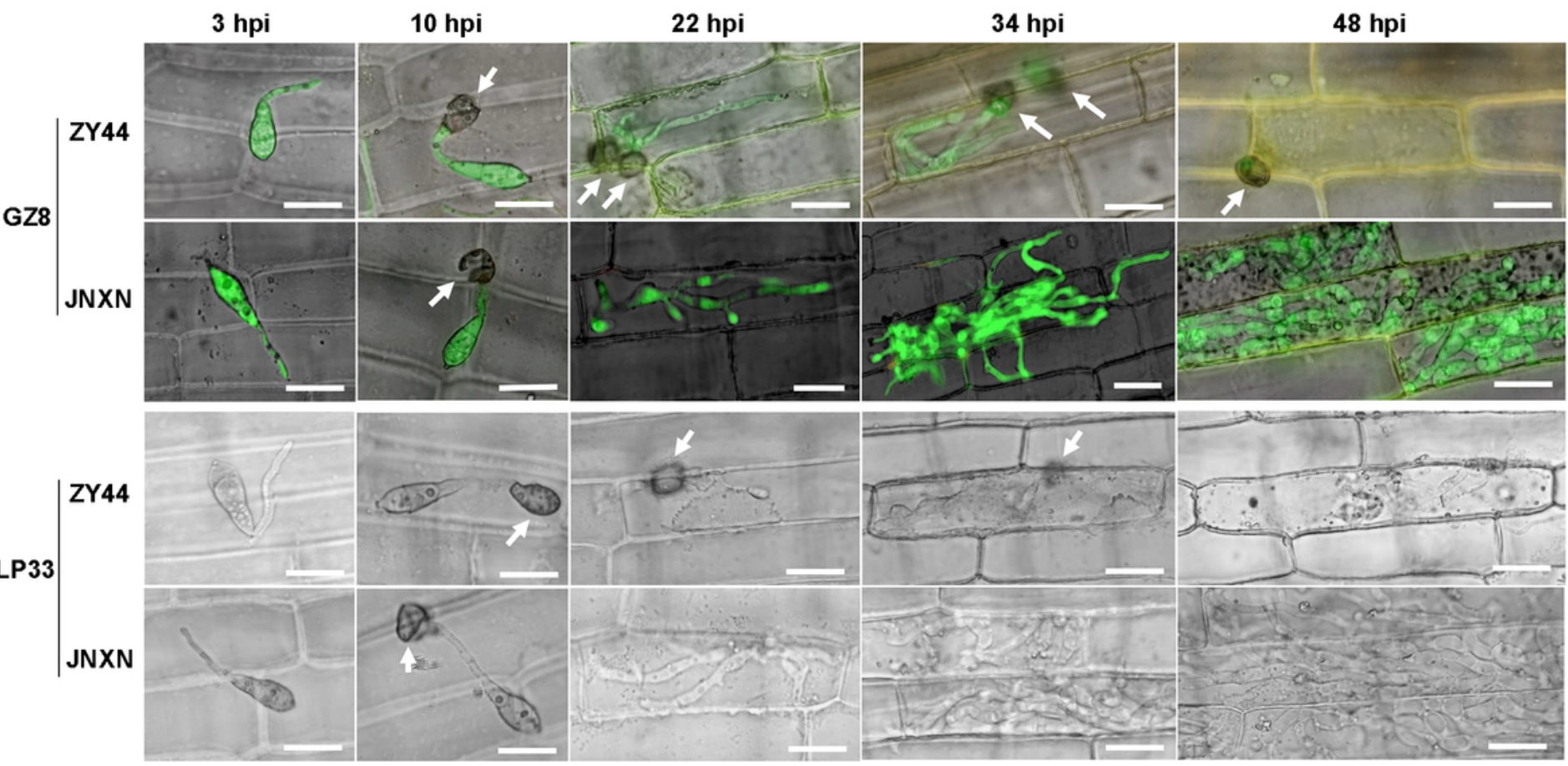

b

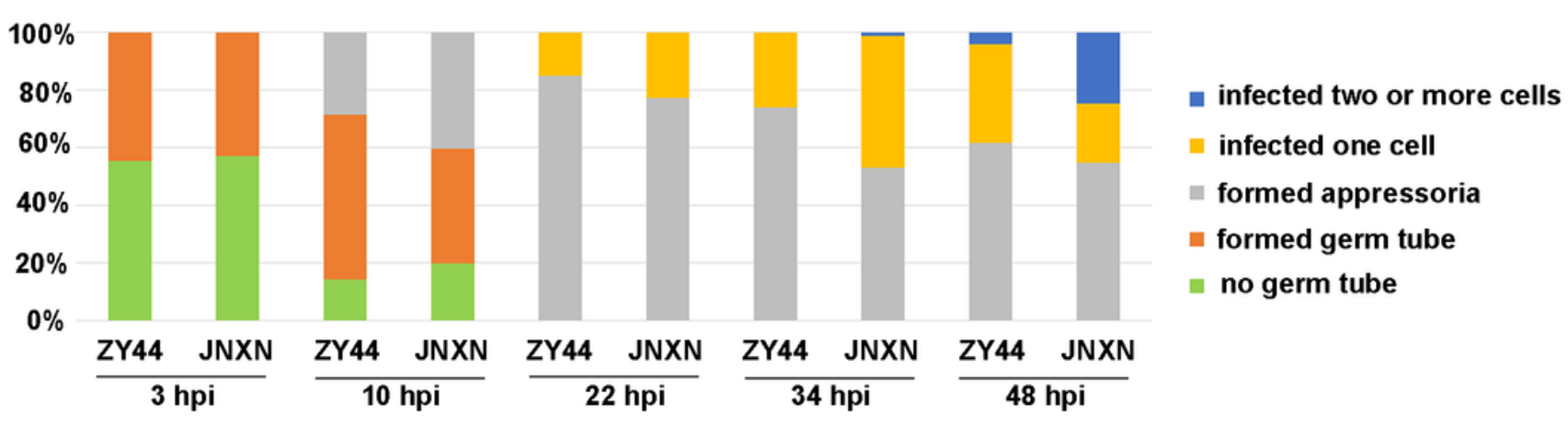

\section{Figure 1}

Microscopic analysis of early infection events in sheaths of Ziyu44 (ZY44) and JNXN inoculated with M. oryzae isolates. (a) Development of M. oryzae on leaf sheaths of resistant rice Ziyu44 and susceptible rice JNXN. Sheaths were inoculated with spore suspensions of M. oryzae Zhong-10-8-14(top) and LP33(bottom), respectively. The inoculated leaf sheaths were examined under a fluorescence microscope 3, 10, 22, 34 and $48 \mathrm{hpi}$, as indicated. Arrows indicate appressorium. (b) Statistics of fungal infection at 3, 10, 22, 34, and $48 \mathrm{hpi}$. At least 100 single-cell interaction sites were examined per time point. The scale bar is $20 \mu \mathrm{m}$ 


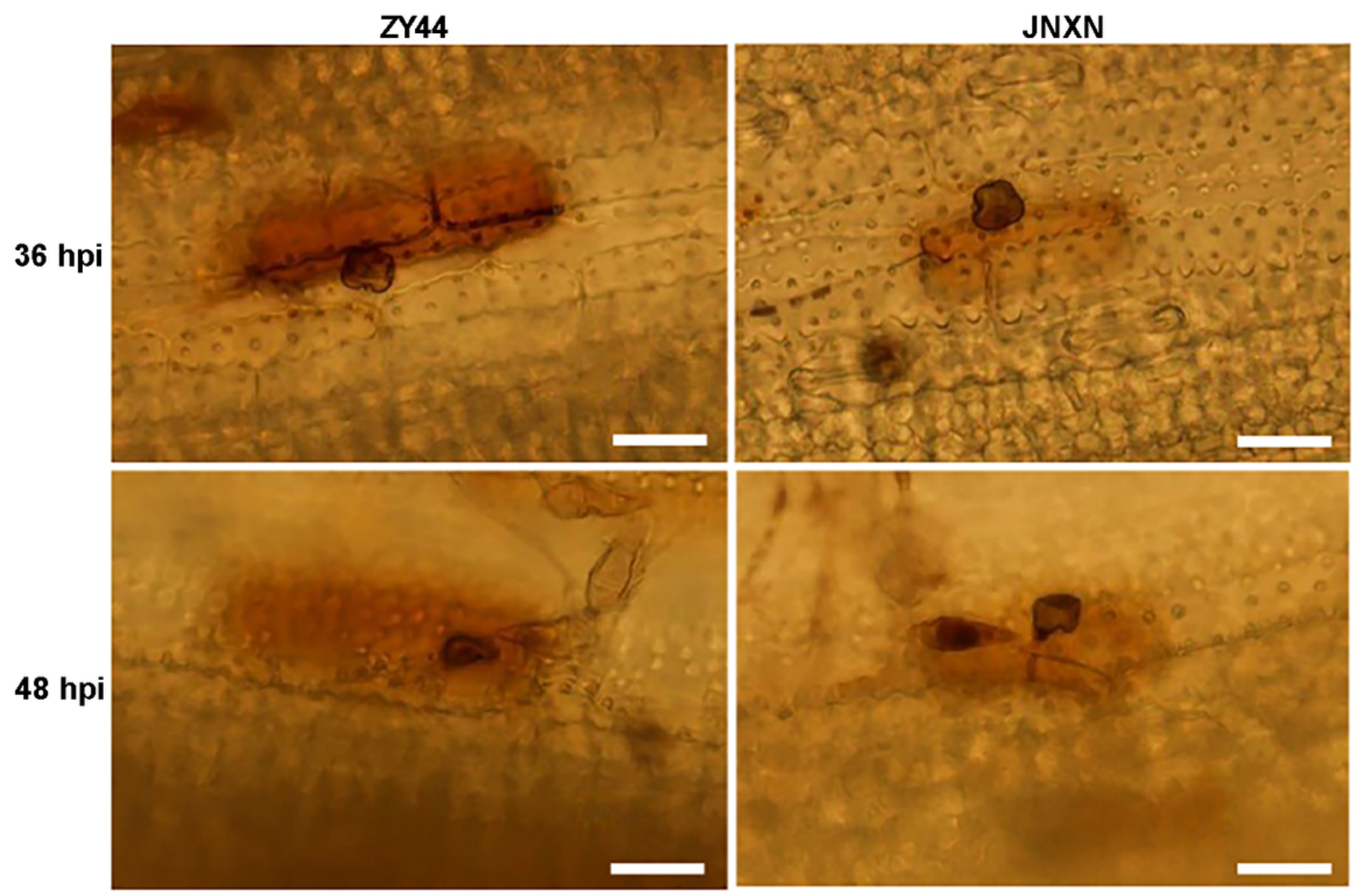

Figure 2

DAB staining at infection sites of Ziyu44 (ZY44) and JNXN leaves. Tawny shading indicates accumulation of $\mathrm{H} 2 \mathrm{O} 2$. The scale bar is $20 \mu \mathrm{m}$

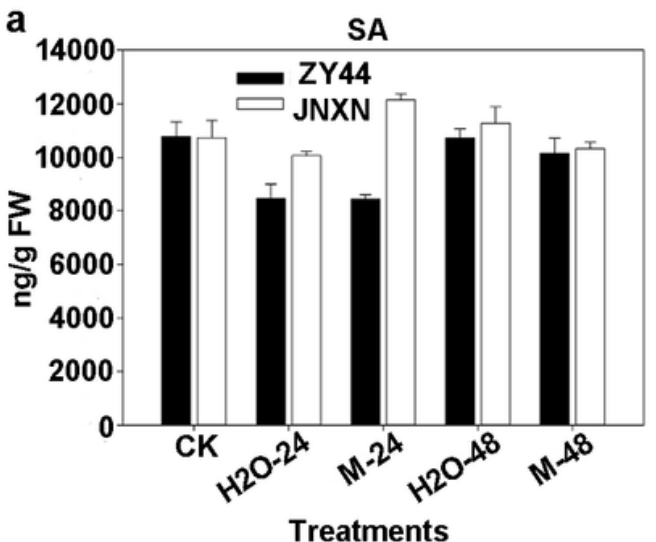

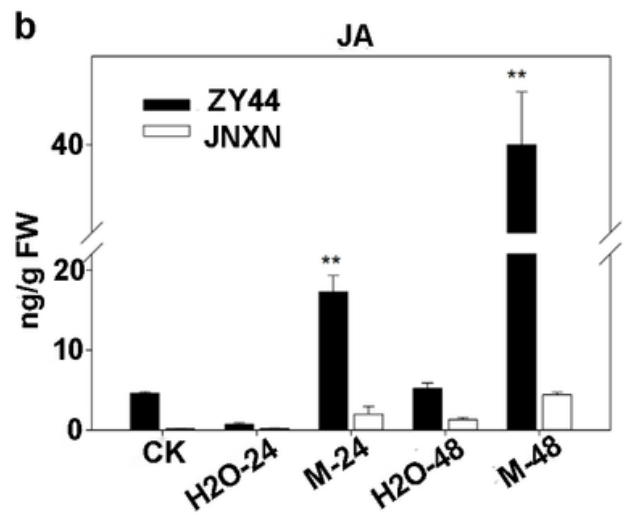

Treatments

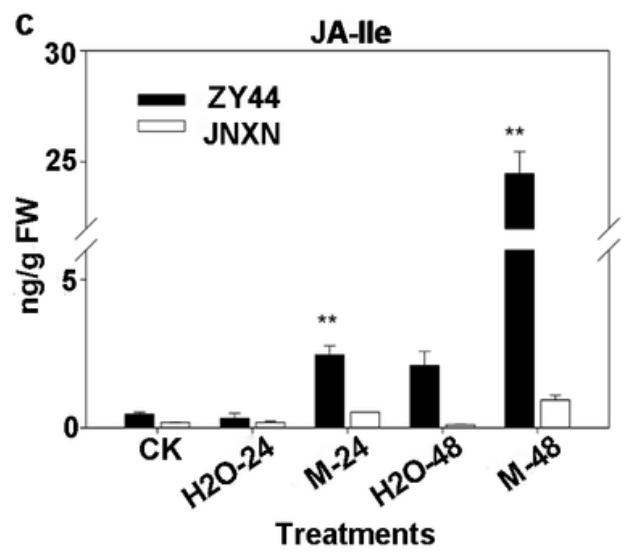

Treatments

\section{Figure 3}

The levels of endogenous SA, JA and JA-lle in Ziyu44 (ZY44) and JNXN plants upon M. oryzae infection. $\mathrm{CK}$, untreated control, $\mathrm{H} 2 \mathrm{O}-24$, inoculated with sterile water at $24 \mathrm{hpi}, \mathrm{M}-24$, inoculated with M. oryzae 
LP33 at 24 hpi, H20-48, inoculated with sterile water at 48 hpi, M-48, inoculated with M. oryzae LP33 at 48 hpi.

a

OsPAL1

b

OsAOS2
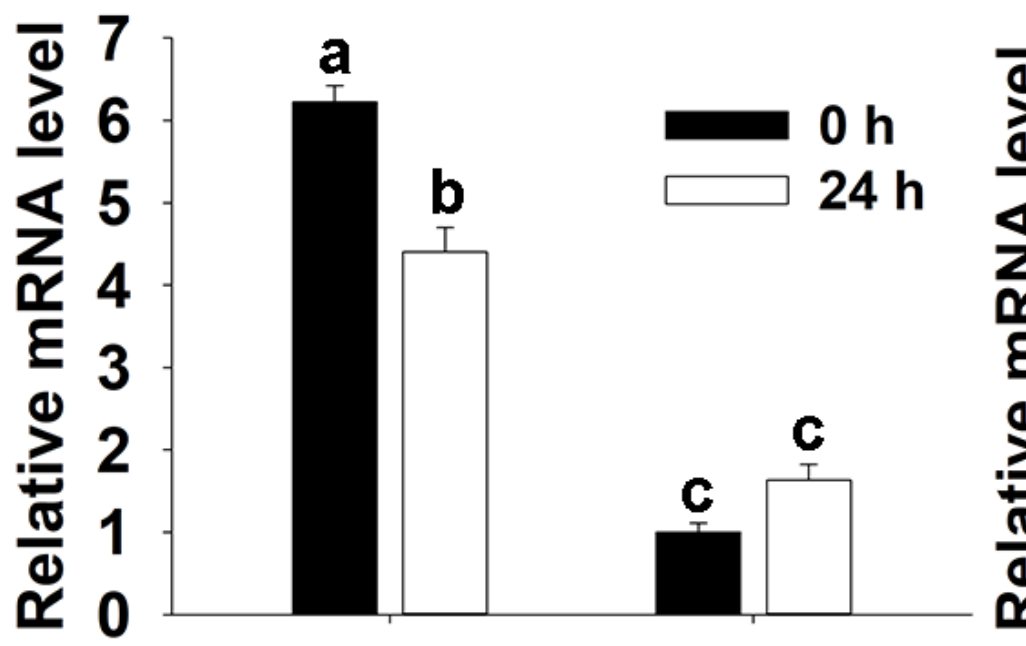

ZY44

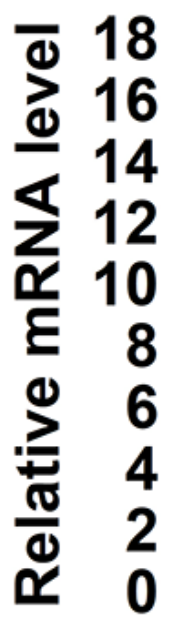

JNXN
18
16
14
12
10
8
6
4
2
0

ZY44 a

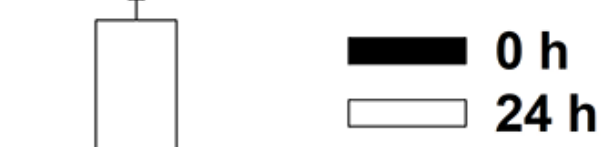

$24 \mathrm{~h}$

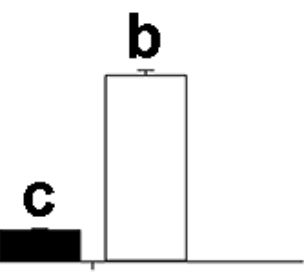

JNXN

Figure 4

Expression of genes involving in SA and JA biosynthesis in Ziyu44 (ZY44) and JNXN inoculated with M. oryzea LP33. Different letters above the bars indicate significant differences as indicated by ANOVA(P < 0.05). Error bars represent mean \pm SD of three independent repests.
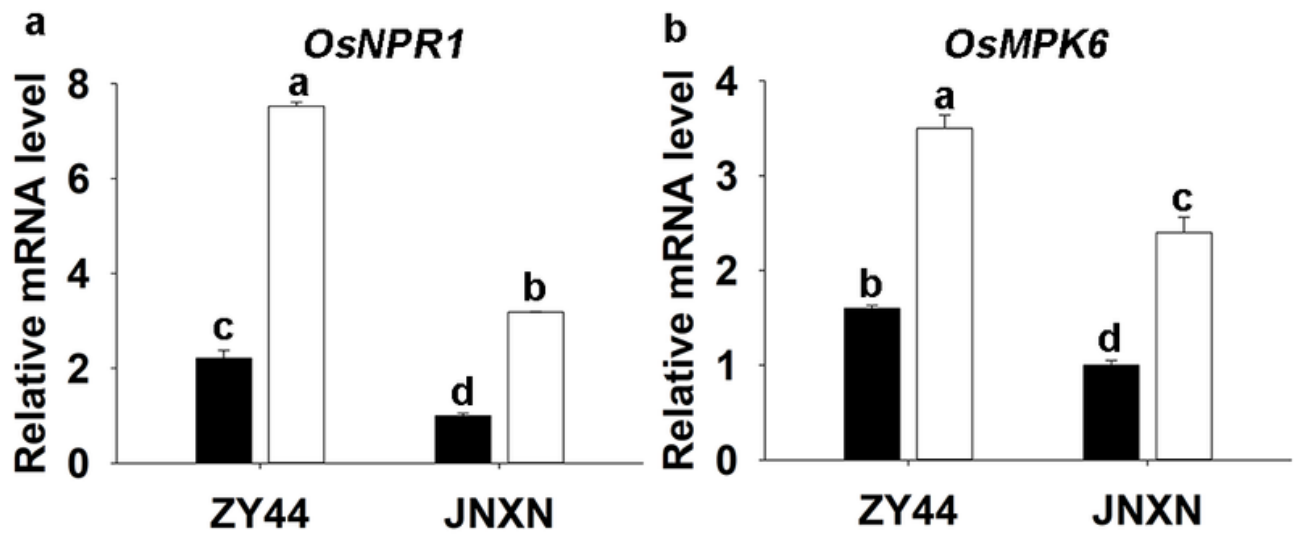

C

OsCOI1b
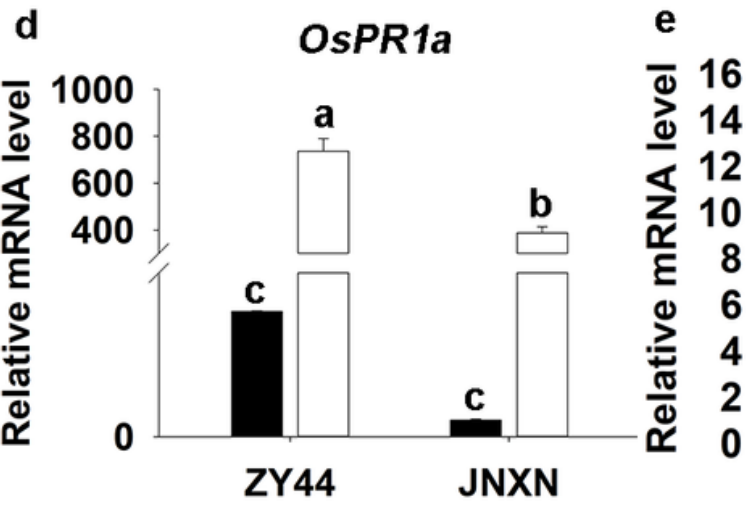

OsPR10a

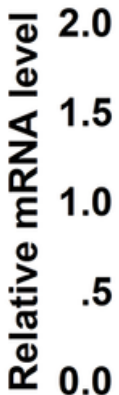

f

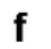

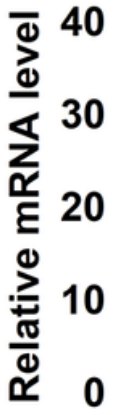

JNXN

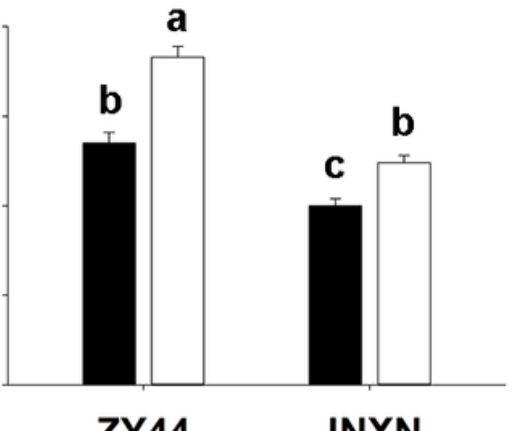

ZY44 JNXN

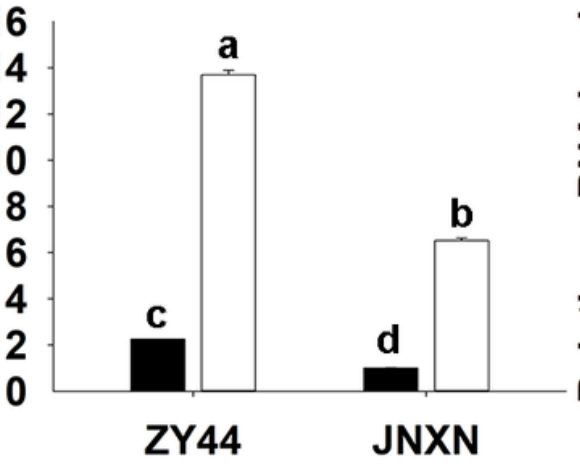




\section{Figure 5}

Expression of pathogenesis-related genes in Ziyu44 (ZY44) and JNXN inoculated with M. oryzea LP33. Different letters above the bars indicatesignificant differences as indicated by ANOVA $(P<0.05)$. Error bars represent mean \pm SD of three independent repests. 